\title{
Single Timescale Regularized Stochastic Approximation Schemes for Monotone Nash games under Uncertainty
}

\author{
Jayash Koshal, Angelia Nedić and Uday V. Shanbhag
}

\begin{abstract}
In this paper, we consider the distributed computation of equilibria arising in monotone stochastic Nash games over continuous strategy sets. Such games arise in settings when the gradient map of the player objectives is a monotone mapping over the cartesian product of strategy sets, leading to a monotone stochastic variational inequality. We consider the application of projection-based stochastic approximation schemes. However, such techniques are characterized by a key shortcoming: they can accommodate strongly monotone mappings only. In fact, standard extensions of stochastic approximation schemes for merely monotone mappings require the solution of a sequence of related strongly monotone problems, a natively two-timescale scheme. Accordingly, we consider the development of single timescale techniques for computing equilibria when the associated gradient map does not admit strong monotonicity. We first show that, under suitable assumptions, standard projection schemes can indeed be extended to allow for strict, rather than strong monotonicity. Furthermore, we introduce a class of regularized stochastic approximation schemes, in which the regularization parameter is updated at every step, leading to a single timescale method. The scheme is a stochastic extension of an iterative Tikhonov regularization method and its global convergence is established. To aid in networked implementations, we consider an extension to this result where players are allowed to choose their steplengths independently and show if the deviation across their choices is suitably constrained, then the convergence of the scheme may be claimed.
\end{abstract}

\section{INTRODUCTION}

This paper considers a class of stochastic Nash games over continuous strategy sets. We cast such a problem as a stochastic variational inequality, for which we consider projection-based stochastic approximation algorithms. Such schemes have been recently employed for the solution of stochastic variational inequalities [12] with strongly monotone and co-coercive maps, which to the best of our knowledge, appears to be the only existing work considering stochastic approximation methods for variational inequalities. The use of stochastic approximation methods has a long tradition in stochastic optimization for both differentiable and nondifferentiable problems, starting with the work of Robbins and Monro [20] for differentiable problems and Ermoliev [8]-[10] for nondifferentiable problems while a subset of more recent efforts include [4], [6], [18].

The typical stochastic approximation procedure, first introduced by Robbins and Monro, works toward finding an extremum of a function $h$ using the following iterative

Department of Industrial and Enterprise Systems Engineering, University of Illinois, Urbana IL 61801, Email: \{koshal1,angelia,udaybag\}@illinois.edu. This work has been supported by NSF CMMI 09-48905 EAGER ARRA award. scheme:

$$
x^{k+1}=x^{k}+a_{k}\left(\nabla h\left(x^{k}\right)+M^{k+1}\right),
$$

where $M^{k+1}$ is a martingale difference sequence. Under reasonable assumptions on the stochastic errors $M^{k}$, stochastic approximation schemes ensure that $\left\{x^{k}\right\}$ converges almost surely to an optimal solution of the problem. Jiang and $\mathrm{Xu}$ [12] consider the use of stochastic approximation for strongly monotone and Lipschitz continuous maps in the realm of stochastic variational inequalities, rather than optimization problems.

In the current work, we consider two schemes that do not require strong monotonicity of the map, a stochastic version of the standard projection method and an iterative Tikhonov regularization method. We first establish almostsure convergence for the stochastic projection method when applied to a stochastic VI with a strictly monotone mapping. To further relax the condition of strict monotonicity, we consider a stochastic iterative Tikhonov regularization method, and we show the almost sure convergence of the method to the minimum norm solution. This result requires users (players) of the associated game to use equal stepsizes. We then investigate the case when users may independently select stepsizes. We show that in this case, the convergence property of the algorithm is preserved under a restriction on the maximum deviation across stepsizes.

It should be remarked that Tikhonov-based regularization methods have a long history in the solution of ill-posed optimization and variational problems [11], [17]. Such schemes, in general, require the solution of regularized (well-posed) problems, each of which may require solution via an iterative process. An alternative lies in updating the regularization parameter at every step, an approach popularly referred to as iterative regularization. While there have been efforts to use such techniques for optimization problems (cf. [17]), there has been noticeably less in the realm of variational inequalities, barring [13] and more recently in [21].

Game-theoretic models find application in a range of settings ranging from wireline and wireless communication networks [3], bandwidth allocation [1], [2], [7], spectrum allocation in radio networks [5] and optical networks [14], [15]. Distributed schemes for computing equilibria have received significant attention recently [1], [2], [14], [16], [19]. Of particular relevance to this paper is that of Pavel and her coauthors [14], [16], the latter in particular employs an extragradient scheme [11] in a deterministic regime, capable of accommodating monotone Nash games. 
A remark is in order regarding our proposed scheme. Our goal lies in developing single timescale gradient-based distributed schemes characterized by low complexity. Such schemes lie at one end of the spectrum of best-response methods, where a gradient step is taken towards obtaining a best-response. While these techniques are not necessarily superior from the standpoint of convergence rate, they remain advantageous from the standpoint of implementability.

The rest of the paper is organized as follows. In Section II, we discuss a $\mathrm{N}$-person stochastic Nash game and its associated equilibrium conditions, as given by a stochastic variational inequality. A projection-based stochastic approximation scheme is presented for this problem in Section III and its convergence is estabilished under an assumption of strict monotonicity of the mapping. This assumption is weakened to monotonicity in Section IV where we propose and analyze a single timescale stochastic iterative Tikhonov regularization method. Convergence of the resulting sequence of iterates is proved in an almost-sure sense, in a regime where players shared the same steplengths and in one where there the steplengths require limited coordination but are not necessarily identical across players. Finally, in Section V, we provide some concluding remarks.

Throughout this paper, we view vectors as columns and write $x^{T}$ to denote the transpose of a vector $x$, and $x^{T} y$ to denote the inner product of vectors $x$ and $y$. We use $\|x\|$ to denote the Euclidean norm of a vector $x$, i.e., $\|x\|=\sqrt{x^{T} x}$. We use $\Pi_{X}$ to denote the Euclidean projection operator onto a set $X$, i.e., $\Pi_{X}(x) \triangleq \operatorname{argmin}_{z \in X}\|x-z\|$. The expectation of a random variable $V$ is denoted by $\mathbb{E}[V]$. Finally, we often use a.s. for almost surely.

\section{PRoblem Description}

We consider an $N$-person stochastic Nash game in which the $i$ th agent solves the parameterized problem

$$
\begin{aligned}
\operatorname{minimize} & \mathbb{E}\left[f_{i}\left(x_{i}, x_{-i}, \xi_{i}\right)\right] \\
\text { subject to } & x_{i} \in K_{i},
\end{aligned}
$$

where $x_{-i}$ denotes the collection $\left\{x_{j}, j \neq i\right\}$ of decisions of all players $j$ other than player $i$. For each $i$, the variable $\xi_{i}$ is random with $\xi_{i}: \Omega_{i} \rightarrow \mathbb{R}^{n_{i}}$, and the function $\mathbb{E}\left[f_{i}\left(x_{i}, x_{-i}, \xi_{i}\right)\right]$ is convex in $x_{i}$ for all $x_{-i} \in \prod_{j \neq i} K_{j}$.

For every $i$, the set $K_{i} \subseteq \mathbb{R}^{n_{i}}$ is a closed convex set. The equilibrium conditions of the game, denoted by $\mathcal{G}$, can be characterized by scalar variational inequality problem denoted by $\operatorname{VI}(K, F)$ where

$$
F(x) \triangleq\left(\begin{array}{c}
\nabla_{x_{1}} \mathbb{E}\left[f_{1}\left(x, \xi_{1}\right)\right] \\
\vdots \\
\nabla_{x_{N}} \mathbb{E}\left[f_{N}\left(x, \xi_{N}\right)\right]
\end{array}\right), \quad K=\prod_{i=1}^{N} K_{i},
$$

with $x \triangleq\left(x_{1}, \ldots, x_{N}\right)^{T}$ and $x_{i} \in K_{i}$ for $i=1, \ldots, N$. Recall that $\operatorname{VI}(K, F)$ requires determining a vector $x^{*} \in K$ such that

$$
\left(x-x^{*}\right)^{T} F\left(x^{*}\right) \geq 0 \quad \text { for all } x \in K .
$$

We let $n=\sum_{i=1}^{N} n_{i}$, and note that the set $K$ is closed and convex set in $\mathbb{R}^{n}$, whenever the sets $K_{i}$ are closed and convex, for $i=1, \ldots, N$. The mapping $F$ maps $x \in K$ to $F(x) \in \mathbb{R}^{n}$.

Standard deterministic algorithms for obtaining solutions to a variational inequality $\mathrm{VI}(K, F)$ require an analytical form for the gradient of the expected-value function. Yet, when the expectation is over a general measure space, analytical forms of the expectation are often hard to obtain. In such settings, stochastic approximation schemes assume relevance. In the remainder of this section, we describe the basic framework of stochastic approximation and the supporting convergence results.

Consider the Robbins-Monro stochastic approximation scheme for solving the stochastic variational inequality $\mathrm{VI}(K, F)$ in (2)-(3), given by

$$
x^{k+1}=\Pi_{K}\left[x^{k}-\alpha_{k}\left(F\left(x^{k}\right)+w^{k}\right)\right] \text { for } k \geq 0,
$$

where $x^{0} \in K$ is an initial point, $F\left(x^{k}\right)$ is the true value of $F(x)$ at $x=x^{k}, \alpha_{k}$ is the step-size, $w^{k}=-F\left(x^{k}\right)+$ $\tilde{F}\left(x^{k}, \xi_{i}^{k}\right)$ is a stochastic error,

$$
\tilde{F}\left(x^{k}, \xi^{k}\right) \triangleq\left(\begin{array}{c}
\nabla_{x_{1}} f_{1}\left(x^{k}, \xi_{1}^{k}\right) \\
\vdots \\
\nabla_{x_{N}} f_{N}\left(x^{k}, \xi_{N}^{k}\right)
\end{array}\right) \text { and } \xi^{k} \triangleq\left(\begin{array}{c}
\xi_{1}^{k} \\
\vdots \\
\xi_{N}^{k}
\end{array}\right) .
$$

The projection scheme (4) is shown to be convergent when the mapping $F$ is strongly monotone and Lipschitz continuous in [12]. In the sequel, we examine how the use of regularization methods can alleviate the need for the strong monotonicity requirement. In fact, we show that either strict monotonicity or mere monotonicity albeit in a regularized setting often suffices.

In our analysis we use some well-known results on supermartingale convergence, which we provide for convenience. The following result is from [17], Lemma 10, page 49.

Lemma 1: Let $V_{k}$ be a sequence of non-negative random variables adapted to $\sigma$-algebra $\mathcal{F}_{k}$ and such that almost surely

$$
\mathbb{E}\left[V_{k+1} \mid \mathcal{F}_{k}\right] \leq\left(1-u_{k}\right) V_{k}+\beta_{k} \quad \text { for all } k \geq 0,
$$

where $0 \leq u_{k} \leq 1, \beta_{k} \geq 0$, and

$$
\sum_{k=0}^{\infty} u_{k}=\infty, \quad \sum_{k=0}^{\infty} \beta_{k}<\infty, \quad \frac{\beta_{k}}{u_{k}} \rightarrow 0 .
$$

Then, $V_{k} \rightarrow 0$ a.s., $\mathbb{E}\left[V_{k}\right] \rightarrow 0$, and for every $a>0, k>0$,

$$
P\left(V_{j} \leq a \text { for all } j \geq k\right) \geq 1-\frac{1}{a} \mathbb{E}\left[V_{k}+\sum_{i=k}^{\infty} \beta_{i}\right] .
$$

The result of the following lemma can be found in [17], Lemma 11, page 50 .

Lemma 2: Let $V_{k}, u_{k}, \beta_{k}$ and $\gamma_{k}$ be non-negative random variables adapted to $\mathcal{F}_{k}$. If almost surely $\sum_{k=1}^{\infty} u_{k}<\infty$, $\sum_{k=1}^{\infty} \beta_{k}<\infty$, and

$$
\mathbb{E}\left[V_{k+1} \mid \mathcal{F}_{k}\right] \leq\left(1+u_{k}\right) V_{k}-\gamma_{k}+\beta_{k} \quad \text { for all } k \geq 0,
$$

then almost surely $\left\{V_{k}\right\}$ is convergent and $\sum_{k=1}^{\infty} \gamma_{k}<\infty$. 


\section{STANDARD PROJECTION SCHEMES UNDER STRICT MONOTONICITY}

Here, we consider standard projection algorithm (4) for a variational inequality $\mathrm{VI}(K, F)$ with a strictly monotone mapping $F$, i.e., a mapping $F$ such that

$$
(F(x)-F(y))^{T}(x-y)>0 \text { for all } x, y \in K, x \neq y,
$$

with equality holding in the preceding relation only when $x=y$. In addition to this, we make some assumptions on the steplength sequence $\left\{\alpha_{k}\right\}$ and the stochastic errors $w^{k}$ that are standard in stochastic approximation schemes.

Throughout the rest of the paper, we use $\mathcal{F}_{k}$ to denote the sigma-field generated by the initial point $x^{0}$ and errors $w^{\ell}$ for $\ell=0,1, \ldots, k$, i.e.,

$$
\mathcal{F}_{k}=\left\{x^{0},\left(w^{\ell}, \ell=0,1, \ldots, k\right)\right\} \quad \text { for } k \geq 0 .
$$

Now, we specify our assumptions for $\operatorname{VI}(K, F)$ in (2)-(3) and the algorithm (4).

Assumption 1 (A1): Let the following hold:

(a) The sets $K_{i} \subseteq \mathbb{R}^{n_{i}}$ are closed and convex;

(b) The mapping $F: K \rightarrow \mathbb{R}^{n}$ is strictly monotone and Lipschitz with constant $L$ over the set $K$;

(c) The steplength satisfies $\alpha_{k}>0$ for all $k, \sum \alpha_{k}=\infty$ and $\sum \alpha_{k}^{2}<\infty$;

(d) The stochastic error is such that $\mathbb{E}\left[w^{k} \mid \mathcal{F}_{k}\right]=0$ and $\sum_{k=1}^{\infty} \alpha_{k}^{2} \mathbb{E}\left[\left\|w^{k}\right\|^{2} \mid \mathcal{F}_{k}\right]<\infty$ a.s.

Since the map $F$ is strictly monotone, the solution to $\operatorname{VI}(K, F)$ is unique whenever it exists (see 2.3.3 Theorem in [11]). Note, however, that the strict monotonicity of $F$ is not enough to guarantee the existence of a solution when the set $K$ is closed and convex.

Next we provide a convergence result for the algorithm (4).

Proposition 1: Let Assumption (A1) hold, and assume that $\operatorname{VI}(K, F)$ has a solution. Let $\left\{x^{k}\right\}$ be the sequence generated by (4). Then, $\left\{x^{k}\right\}$ converges to the solution $x^{*}$ of $\mathrm{VI}(K, F)$ almost surely.

Proof: As discussed earlier, in view of the strict monotonicity of $F$, the solution $x^{*}$ of $\operatorname{VI}(K, F)$ is unique. Furthermore, we have $x^{*}=\Pi_{K}\left[x^{*}-\alpha_{k} F\left(x^{*}\right)\right]$, which together with the non-expansiveness property of the projection operator yields

$$
\begin{aligned}
& \left\|x^{k+1}-x^{*}\right\|^{2} \leq\left\|x^{k}-x^{*}-\alpha_{k}\left(F\left(x^{k}\right)-F\left(x^{*}\right)+w^{k}\right)\right\|^{2} \\
& =\left\|x^{k}-x^{*}\right\|^{2}+\alpha_{k}^{2}\left\|F\left(x^{k}\right)-F\left(x^{*}\right)\right\|^{2}+\alpha_{k}^{2}\left\|w^{k}\right\|^{2} \\
& -2 \alpha_{k}\left(x^{k}-x^{*}\right)^{T}\left(F\left(x^{k}\right)-F\left(x^{*}\right)\right)-2 \alpha_{k}\left(x^{k}-x^{*}\right)^{T} w^{k} \\
& -2 \alpha_{k}\left(F\left(x^{k}\right)-F\left(x^{*}\right)\right)^{T} w^{k} .
\end{aligned}
$$

By taking expectation and using $\mathbb{E}\left[w^{k} \mid \mathcal{F}_{k}\right]=0$ and Lipschitz continuity of mapping $F$, we obtain

$$
\begin{aligned}
& \mathbb{E}\left[\left\|x^{k+1}-x^{*}\right\|^{2} \mid \mathcal{F}_{k}\right] \leq\left(1+\alpha_{k}^{2} L^{2}\right)\left\|x^{k}-x^{*}\right\|^{2} \\
& \quad+\alpha_{k}^{2} \mathbb{E}\left[\left\|w^{k}\right\|^{2} \mid \mathcal{F}_{k}\right]-2 \alpha_{k}\left(x^{k}-x^{*}\right)^{T}\left(F\left(x^{k}\right)-F\left(x^{*}\right)\right),
\end{aligned}
$$

which can be compactly written as

$$
\mathbb{E}\left[\left\|x^{k+1}-x^{*}\right\|^{2} \mid \mathcal{F}_{k}\right] \leq\left(1+u_{k}\right)\left\|x^{k}-x^{*}\right\|^{2}-\gamma_{k}+\beta_{k},
$$

where $u_{k}=\alpha_{k}^{2} L^{2}, \beta_{k}=\alpha_{k}^{2} \mathbb{E}\left[\left\|w^{k}\right\|^{2} \mid \mathcal{F}_{k}\right]$, and $\gamma_{k}=$ $2 \alpha_{k}\left(x^{k}-x^{*}\right)^{T}\left(F\left(x^{k}\right)-F\left(x^{*}\right)\right)$. By Assumptions (A1c) and (A1d), we have $\sum_{k=1}^{\infty} u_{k}<\infty$ and $\sum_{k=1}^{\infty} \beta_{k}<\infty$. It follows from Lemma 2 that $\left\|x^{k}-x^{*}\right\|$ converges almost surely to a limit point $\bar{v} \geq 0$ and $\sum_{k=1}^{\infty} \gamma_{k}<\infty$ almost surely.

It remains to show that $\bar{v} \equiv 0$, which is equivalent to $x_{k} \rightarrow x^{*}$ almost surely. Since we have

$$
\sum_{k=1}^{\infty} 2 \alpha_{k}\left(x^{k}-x^{*}\right)^{T}\left(F\left(x^{k}\right)-F\left(x^{*}\right)\right)<\infty \quad \text { a.s. }
$$

and $\sum_{k=1}^{\infty} \alpha_{k}=\infty$ by Assumption (A1c), it follows that

$$
\liminf _{k \rightarrow \infty}\left(x^{k}-x^{*}\right)^{T}\left(F\left(x^{k}\right)-F\left(x^{*}\right)\right)=0 \quad \text { a.s. }
$$

By the strict monotonicity of $F$, this implies that along a subsequence, we have that $x_{k} \rightarrow x^{*}$ almost surely. This and the relation

$$
\lim _{k \rightarrow \infty}\left\|x_{k}-x^{*}\right\|=\bar{v} \geq 0 \quad \text { a.s. }
$$

imply that $x_{k} \rightarrow x^{*}$ a.s.

We note that if Assumption (A1c) is strengthened to $F$ being strongly monotone, then we immediately have almost sure convergence of the algorithm, which has been established in [12].

\section{Stochastic Iterative Tikhonov Schemes}

In the previous section, single-valuedness of the map $F$ was ensured by a strict monotonicity of $F$. In this section, we relax the strict monotonicity assumption on the mapping, but we perturb the map with a regularizing term, which ensures the single-valuedness of the solution set of the resulting perturbed map. This is a classical approach known as Tikhonov regularization [11, Ch. 12]. In the context of variational inequalities, such techniques typically rely on solving a sequence of strongly monotone problems, denoted by $\mathrm{VI}\left(K, F+\epsilon_{k} y\right)$, allowing us to claim that

$$
\lim _{k \rightarrow \infty} y^{k}=x^{*}
$$

where $y^{k}$ is a solution of $\operatorname{VI}\left(K, F+\epsilon_{k} y\right)$ and $x^{*}$ is the least norm solution of $\mathrm{VI}(K, F)$. Thus, to reach a solution of $\operatorname{VI}(K, F)$, one has to solve a sequence of variational inequality problems, namely $\mathrm{VI}\left(K, F+\epsilon_{k} y\right)$ along some sequence $\left\{\epsilon_{k}\right\}$. However, determining a solution $y^{k}$ for a regularized variational inequality $\operatorname{VI}\left(K, F+\epsilon_{k} y\right)$ itself requires an iterative algorithm. Alternatively, one may seek an inexact solution to each $\operatorname{VI}\left(K, F+\epsilon_{k} y\right)$ in constructing such a trajectory [11].

We propose an algorithm that, instead of solving a sequence of variational inequality problems, moves toward the least norm solution by appropriately coordinating the regularization parameters $\epsilon_{k}$ with the steplengths $\alpha_{k}$. Specifically, our intent lies in developing algorithms that are characterized by (1) a single-timescale structure; (2) a distributed architecture that can accommodate computation of equilibria; and (3) finally, accommodate uncertainty via an expected-value 
objectives. The resulting coupled agent-specific Tikhonov updates are given by: for $i=1, \ldots, N$,

$$
x_{i}^{k+1}=\Pi_{K_{i}}\left[x_{i}^{k}-\alpha_{k}\left(F_{i}\left(x^{k}\right)+\epsilon_{k} x_{i}^{k}\right)+w_{i}^{k}\right],
$$

where $x_{i}^{0} \in K_{i}$ is initial point, $F_{i}\left(x^{k}\right)$ denotes the $i$ th component of mapping $F$ evaluated at $x^{k}, w_{i}^{k}$ is the $i$ th component of stochastic error vector $w^{k}$ and $\alpha_{k}$ is the stepsize chosen at the $k$ th iterate. Note that, as in Section III, the iterate updates can be compactly written as

$$
x^{k+1}=\Pi_{K}\left[x_{i}^{k}-\alpha_{k}\left(F\left(x^{k}\right)+\epsilon_{k} x^{k}\right)+w^{k}\right],
$$

where $F, K$ and $w^{k}$ are as in Section III.

In Section IV-A, we examine the global convergence of the distributed regularized scheme given by (5). A requirement of this scheme is that $\alpha_{k}$ be enforced uniformly across all agents. We weaken such a requirement in Section IV-B and provide a framework under which players are accorded some flexibility in choosing steplengths.

\section{A. Coordinated Steplengths}

We let $\operatorname{SOL}(K, F)$ denote the solution set of $\operatorname{VI}(K, F)$ in (2)-(3), and $y^{k}$ be the solution to $\operatorname{VI}\left(K, F+\epsilon_{k} y\right)$. We have the following result for the Tikhonov sequence $\left\{y^{k}\right\}$.

Lemma 3: Let the set $K \subseteq \mathbb{R}^{n}$ be closed and convex, and let the map $F: K \rightarrow \mathbb{R}^{n}$ be continuous and monotone over $K$. Assume that $\operatorname{SOL}(K, F)$ is nonempty. Consider a standard Tikhonov scheme for $\operatorname{VI}(K, F)$, where $\left\{y^{k}\right\}$ is the sequence of exact solutions to $\operatorname{VI}\left(K, F+\epsilon_{k} y\right), k \geq 0$. Then

$$
\left\|y^{k}-y^{k-1}\right\| \leq M_{y} \frac{\left|\epsilon_{k-1}-\epsilon_{k}\right|}{\epsilon_{k}} \quad \text { for all } k \geq 1,
$$

where $M_{y}$ is a norm bound on the Tikhonov sequence, i.e., $\left\|y^{k}\right\| \leq M_{y}$ for all $k \geq 0$.

Proof: Since $\operatorname{SOL}(K, F) \neq \emptyset$, the Tikhonov sequence converges to the least norm solution of $\mathrm{VI}(K, F)$ (cf. [11], 12.2.3 Theorem, page 1128), i.e.,

$$
\lim _{k \rightarrow \infty} y^{k}=x^{*}
$$

where $x^{*}$ is the least norm solution of $\operatorname{VI}(K, F)$. Since $y^{k}$ solves $\operatorname{VI}\left(K, F+\epsilon_{k} y\right)$ for each $k \geq 0$, we have for $k \geq 1$,

$$
\begin{aligned}
\left(y^{k-1}-y^{k}\right)^{T}\left(F\left(y^{k}\right)+\epsilon_{k} y^{k}\right) & \geq 0, \\
\left(y^{k}-y^{k-1}\right)^{T}\left(F\left(y^{k-1}\right)+\epsilon_{k-1} y^{k-1}\right) & \geq 0 .
\end{aligned}
$$

By adding the preceding relations, we obtain

$$
\begin{aligned}
& \left(y^{k-1}-y^{k}\right)^{T}\left(F\left(y^{k}\right)-F\left(y^{k-1}\right)\right) \\
& +\left(y^{k-1}-y^{k}\right)^{T}\left(\epsilon_{k} y^{k}-\epsilon_{k-1} y^{k-1}\right) \geq 0 .
\end{aligned}
$$

By the monotonicity of the mapping $F$, it follows

$$
\left(y^{k-1}-y^{k}\right)^{T}\left(\epsilon_{k} y^{k}-\epsilon_{k-1} y^{k-1}\right) \geq 0
$$

and thus

$$
\left(y^{k-1}-y^{k}\right)^{T}\left(\epsilon_{k} y^{k}-\epsilon_{k} y^{k-1}+\epsilon_{k} y^{k-1}-\epsilon_{k-1} y^{k-1}\right) \geq 0 .
$$

By rearranging the terms in the above expression, we obtain

$$
\begin{aligned}
\left(y^{k-1}-y^{k}\right)^{T}\left(\epsilon_{k}-\epsilon_{k-1}\right) y^{k-1} & \geq \epsilon_{k}\left(y^{k-1}-y^{k}\right)^{T}\left(y^{k-1}-y^{k}\right) \\
& =\epsilon_{k}\left\|y^{k}-y^{k-1}\right\|^{2} .
\end{aligned}
$$

However, through an application of Cauchy-Schwartz inequality, the left hand side is bounded from above as

$\left(y^{k-1}-y^{k}\right)^{T}\left(\epsilon_{k}-\epsilon_{k-1}\right) y^{k-1} \leq \| y^{k-1}-y^{k}|||| y^{k-1}|| \epsilon_{k-1}-\epsilon_{k} \mid$.

Combining the preceding relations we obtain

$$
\left\|y^{k}-y^{k-1}\right\| \leq\left\|y^{k-1}\right\| \frac{\left|\epsilon_{k-1}-\epsilon_{k}\right|}{\epsilon_{k}} \leq M_{y} \frac{\left|\epsilon_{k-1}-\epsilon_{k}\right|}{\epsilon_{k}},
$$

where $\left\|y^{k}\right\| \leq M_{y}$ for all $k$, which follows from the boundedness of Tikhonov sequence.

We do not rely on the strict monotonicity assumption on mapping $F$, but impose the following.

Assumption 2 (A2): Let the following hold:

(a) The sets $K_{i} \subseteq \mathbb{R}^{n_{i}}$ are closed and convex for all $i$;

(b) The mapping $F: K \rightarrow \mathbb{R}^{n}$ is monotone and Lipschitz with constant $L$ over the set $K$;

(c) The stochastic error is such that $\mathbb{E}\left[w^{k} \mid \mathcal{F}_{k}\right]=0$ and $\sum_{k=1}^{\infty} \alpha_{k}^{2} \mathbb{E}\left[\left\|w^{k}\right\|^{2} \mid \mathcal{F}_{k}\right]<\infty$ a.s.

We next state the assumptions that the regularization parameter $\left\{\epsilon_{k}\right\}$ and the stepsize sequence $\left\{\alpha_{k}\right\}$ satisfy for the remainder of this subsection. We assume that the parameters $\epsilon_{k}$ are such that $\epsilon_{k} \leq \epsilon_{0}$ for all $k$. We also assume the following.

Assumption 3 (A3): Let the following hold:

(a) $\frac{\alpha_{k}}{\epsilon_{k}}\left(\epsilon_{0}+L\right)^{2} \leq c$ for all $k$, where $c \in(0,1)$;

(b) $0<\alpha_{k} \epsilon_{k} \leq \frac{1}{1-c}$ for all $k$ and $\lim _{k \rightarrow \infty} \epsilon_{k}=0$;

(c) $\sum_{k=1}^{\infty} \frac{\left(\epsilon_{k-1}-\epsilon_{k}\right)}{\epsilon_{k}^{2}}\left(1+\frac{1}{\alpha_{k} \epsilon_{k}}\right)<\infty$;

(d) $\lim _{k \rightarrow \infty} \frac{\left|\epsilon_{k-1}-\epsilon_{k}\right|}{\epsilon_{k}^{3} \alpha_{k}}\left(1+\frac{1}{\alpha_{k} \epsilon_{k}}\right)=0$;

(e) $\lim _{k \rightarrow \infty} \frac{\alpha_{k}}{\epsilon_{k}} \mathbb{E}\left[\left\|w^{k}\right\|^{2} \mid \mathcal{F}_{k}\right]=0$;

(f) $\sum_{k=1}^{\infty} \alpha_{k} \epsilon_{k}=\infty$.

We now provide an almost sure convergence result for the scheme (5).

Proposition 2: Suppose that (A2) and (A3) hold. Also, assume that $\operatorname{SOL}(K, F)$ is nonempty. Let $\left\{x^{k}\right\}$ be the sequence generated by iterative scheme (5). Then, $\left\{x^{k}\right\}$ converges to the least-norm solution $x^{*}$ of $\mathrm{VI}(K, F)$ almost surely. Also, for $\left\{x^{k}\right\}$ we have

$$
\begin{aligned}
& \text { Prob }\left\{\left\|x^{j}-y^{j-1}\right\|^{2} \leq a \text { for all } j \geq k\right\} \\
& \geq 1-\frac{1}{a} \mathbb{E}\left[\left\|x^{k}-y^{k-1}\right\|^{2} \mid \mathcal{F}_{k}\right] \\
& +\sum_{i=k}^{\infty}\left(q_{k} M_{y}^{2} \frac{\left(\epsilon_{i-1}-\epsilon_{i}\right)^{2}}{\epsilon_{i}^{2}}\left(1+\frac{1}{\alpha_{i} \epsilon_{i}}\right)+\alpha_{i}^{2} \mathbb{E}\left[\left\|w^{i}\right\|^{2} \mid \mathcal{F}_{i}\right]\right)
\end{aligned}
$$

Proof: By using the compact representation of the algorithm given in (6), the relation $y^{k}=\Pi_{K}\left[y^{k}-\alpha_{k}\left(F\left(y^{k}\right)+\right.\right.$ $\left.\left.\epsilon_{k} y^{k}\right)\right]$ and the non-expansive property of the projection operator, the difference $\left\|x^{k+1}-y^{k}\right\|^{2}$ can be expanded as

$$
\begin{aligned}
& \left\|x^{k+1}-y^{k}\right\|^{2} \\
& \leq\left\|\left(x^{k}-y^{k}\right)-\alpha_{k}\left(F\left(x^{k}\right)-F\left(y^{k}\right)+\epsilon_{k}\left(x^{k}-y^{k}\right)+w^{k}\right)\right\|^{2} \\
& =\left\|x^{k}-y^{k}\right\|^{2}-2 \alpha_{k}\left(x^{k}-y^{k}\right)^{T}\left(F\left(x^{k}\right)-F\left(y^{k}\right)\right) \\
& -2 \alpha_{k} \epsilon_{k}\left(x^{k}-y^{k}\right)^{T}\left(x^{k}-y^{k}\right)-2 \alpha_{k}\left(x^{k}-y^{k}\right)^{T} w^{k} \\
& +\alpha_{k}^{2} \underbrace{\left\|F\left(x^{k}\right)-F\left(y^{k}\right)+\epsilon_{k}\left(x^{k}-y^{k}\right)+w^{k}\right\|^{2}}_{\text {Term } 1}
\end{aligned}
$$


From the expression above, Term 1 may be expanded as

$$
\begin{aligned}
& \left\|F\left(x^{k}\right)-F\left(y^{k}\right)+\epsilon_{k}\left(x^{k}-y^{k}\right)+w^{k}\right\|^{2} \\
= & \left\|F\left(x^{k}\right)-F\left(y^{k}\right)\right\|^{2}+\epsilon_{k}^{2}\left\|x^{k}-y^{k}\right\|^{2}+\left\|w^{k}\right\|^{2} \\
+ & 2 \epsilon_{k}\left(x^{k}-y^{k}\right)^{T}\left(F\left(x^{k}\right)-F\left(y^{k}\right)\right) \\
+ & 2\left(\epsilon_{k}\left(x^{k}-y^{k}\right)+\left(F\left(x^{k}\right)-F\left(y^{k}\right)\right)\right)^{T} w^{k} .
\end{aligned}
$$

Taking expectations and combining (7) and (8) with $\mathbb{E}\left[w^{k}\right.$ $\left.\mathcal{F}_{k}\right]=0$, we obtain

$$
\begin{aligned}
& \mathbb{E}\left[\left\|x^{k+1}-y^{k}\right\|^{2} \mid \mathcal{F}_{k}\right] \\
& \leq\left(1-2 \alpha_{k} \epsilon_{k}+\alpha_{k}^{2} \epsilon_{k}^{2}\right)\left\|x^{k}-y^{k}\right\|^{2}+\alpha_{k}^{2}\left\|F\left(x^{k}\right)-F\left(y^{k}\right)\right\|^{2} \\
& +\alpha_{k}^{2} \mathbb{E}\left[\left\|w^{k}\right\|^{2} \mid \mathcal{F}_{k}\right]+2 \alpha_{k}^{2} \epsilon_{k}\left(x^{k}-y^{k}\right)^{T}\left(F\left(x^{k}\right)-F\left(y^{k}\right)\right) \\
& -2 \alpha_{k}\left(x^{k}-y^{k}\right)^{T}\left(F\left(x^{k}\right)-F\left(y^{k}\right)\right) .
\end{aligned}
$$

Since $F$ is monotone the last term is non-positive. Furthermore, by recalling that $F$ is Lipschitz continuous with constant $L$, we have that

$$
\begin{aligned}
& \mathbb{E}\left[\left\|x^{k+1}-y^{k}\right\|^{2} \mid \mathcal{F}_{k}\right] \\
\leq & \left(1-2 \alpha_{k} \epsilon_{k}+\alpha_{k}^{2}\left(L^{2}+2 \epsilon_{k} L+\epsilon_{k}^{2}\right)\right)\left\|x^{k}-y^{k}\right\|^{2} \\
+ & \alpha_{k}^{2} \mathbb{E}\left[\left\|w^{k}\right\|^{2} \mid \mathcal{F}_{k}\right] .
\end{aligned}
$$

Next we relate $\left\|x^{k}-y^{k}\right\|$ and $\left\|x^{k}-y^{k-1}\right\|$. By the triangle inequality $\left\|x^{k}-y^{k}\right\| \leq\left\|x^{k}-y^{k-1}\right\|+\left\|y^{k-1}-y^{k}\right\|$ while from Lemma 3 we have

$$
\left\|y^{k-1}-y^{k}\right\| \leq M_{y} \frac{\left|\epsilon_{k-1}-\epsilon_{k}\right|}{\epsilon_{k}} .
$$

Therefore, it follows that

$$
\begin{aligned}
\left\|x^{k}-y^{k}\right\|^{2} & \leq\left\|x^{k}-y^{k-1}\right\|^{2}+M_{y}^{2} \frac{\left(\epsilon_{k-1}-\epsilon_{k}\right)^{2}}{\epsilon_{k}^{2}} \\
& +2 \frac{\left|\epsilon_{k-1}-\epsilon_{k}\right|}{\epsilon_{k}}\left\|x^{k}-y^{k-1}\right\| M_{y} .
\end{aligned}
$$

The last term can be approximated as

$$
\begin{aligned}
& 2 \frac{\left|\epsilon_{k-1}-\epsilon_{k}\right|}{\epsilon_{k}}\left\|x^{k}-y^{k-1}\right\| M_{y} \\
= & 2 \sqrt{\alpha_{k} \epsilon_{k}}\left\|x^{k}-y^{k-1}\right\| \frac{\left|\epsilon_{k-1}-\epsilon_{k}\right|}{\epsilon_{k} \sqrt{\alpha_{k} \epsilon_{k}}} M_{y} \\
\leq & \alpha_{k} \epsilon_{k}\left\|x^{k}-y^{k-1}\right\|^{2}+\frac{\left(\epsilon_{k-1}-\epsilon_{k}\right)^{2}}{\alpha_{k} \epsilon_{k}^{3}} M_{y}^{2} .
\end{aligned}
$$

Combining the preceding two relations we have

$$
\begin{aligned}
\left\|x^{k}-y^{k}\right\|^{2} & \leq\left(1+\alpha_{k} \epsilon_{k}\right)\left\|x^{k}-y^{k-1}\right\|^{2} \\
& +M_{y}^{2} \frac{\left|\epsilon_{k-1}-\epsilon_{k}\right|^{2}}{\epsilon_{k}^{2}}\left(1+\frac{1}{\alpha_{k} \epsilon_{k}}\right) .
\end{aligned}
$$

Letting $q_{k}=1-2 \alpha_{k} \epsilon_{k}+\alpha_{k}^{2}\left(\epsilon_{k}+L\right)^{2}$ and combining (9) and (11), we have

$$
\begin{aligned}
\mathbb{E}\left[\left\|x^{k+1}-y^{k}\right\|^{2} \mid \mathcal{F}_{k}\right] & \leq q_{k}\left(1+\alpha_{k} \epsilon_{k}\right)\left\|x^{k}-y^{k-1}\right\|^{2} \\
& +q_{k} M_{y}^{2} \frac{\left(\epsilon_{k-1}-\epsilon_{k}\right)^{2}}{\epsilon_{k}^{2}}\left(1+\frac{1}{\alpha_{k} \epsilon_{k}}\right) \\
& +\alpha_{k}^{2} \mathbb{E}\left[\left\|w^{k}\right\|^{2} \mid \mathcal{F}_{k}\right] .
\end{aligned}
$$

Next, we estimate the coefficient of $\left\|x^{k}-y^{k-1}\right\|^{2}$ in the preceding relation. Let $a_{k}:=q_{k}\left(1+\alpha_{k} \epsilon_{k}\right)$ and note that $q_{k}<1$ by (A3a), from which we see that

$$
\begin{aligned}
a_{k} & =q_{k}\left(1+\alpha_{k} \epsilon_{k}\right) \\
& \leq 1-2 \alpha_{k} \epsilon_{k}+\alpha_{k}^{2}\left(\epsilon_{k}+L\right)^{2}+\alpha_{k} \epsilon_{k} \\
& =1-\alpha_{k} \epsilon_{k}\left(1-\frac{\alpha_{k}}{\epsilon_{k}}\left(\epsilon_{k}+L\right)^{2}\right) .
\end{aligned}
$$

Using $\epsilon_{k} \leq \epsilon_{0}$ and $\frac{\alpha_{k}}{\epsilon_{k}}\left(\epsilon_{0}+L\right)^{2} \leq c$, we find

$$
a_{k} \leq 1-\alpha_{k} \epsilon_{k}(1-c),
$$

and the estimate for $\mathbb{E}\left[\left\|x^{k+1}-y^{k}\right\|^{2} \mid \mathcal{F}_{k}\right]$ can be compactly written as:

$$
\mathbb{E}\left[\left\|x^{k+1}-y^{k}\right\|^{2} \mid \mathcal{F}_{k}\right] \leq\left(1-u_{k}\right)\left\|x^{k}-y^{k-1}\right\|^{2}+v_{k},
$$

where $u_{k} \triangleq \alpha_{k} \epsilon_{k}(1-c)$ and

$v_{k} \triangleq q_{k} M_{y}^{2} \frac{\left(\epsilon_{k-1}-\epsilon_{k}\right)^{2}}{\epsilon_{k}^{2}}\left(1+\frac{1}{\alpha_{k} \epsilon_{k}}\right)+\alpha_{k}^{2} \mathbb{E}\left[\left\|w^{k}\right\|^{2} \mid \mathcal{F}_{k}\right]$.

To claim the convergence statement of the sequence $\left\{\left\|x^{k+1}-y^{k}\right\|\right\}$, we need to establish the properties of $u_{k}$ and $v_{k}$.

First, we show that $0 \leq u_{k} \leq 1$ and $\sum_{k=1}^{\infty} u_{k}=\infty$. When $c<1$, we have that $u_{k}=\alpha_{k} \epsilon_{k}(1-c) \geq 0$. Furthermore, from (A3b) it can be easily verified that $u_{k} \leq 1$. Then $\sum_{k=1}^{\infty} u_{k}=\infty$ since $u_{k}=\alpha_{k} \epsilon_{k}(1-c), c<1$ and $\sum_{k=1}^{\infty} \alpha_{k} \epsilon_{k}=\infty$, of which the last holds by (A3f).

Next, we observe that $v_{k} / u_{k} \geq 0$ and it suffices to show that its upper bound converges to zero. Note that, since $q_{k} \leq$ 1 , the ratio $v_{k} / u_{k}$ can be bounded above by

$$
\begin{aligned}
& \leq \frac{1}{\alpha_{k} \epsilon_{k}(1-c)} M_{y}^{2} \frac{\left(\epsilon_{k-1}-\epsilon_{k}\right)^{2}}{\epsilon_{k}^{2}}\left(1+\frac{1}{\alpha_{k} \epsilon_{k}}\right) \\
& +\frac{\alpha_{k}^{2}}{\alpha_{k} \epsilon_{k}(1-c)} \mathbb{E}\left[\left\|w^{k}\right\|^{2} \mid \mathcal{F}_{k}\right] \\
& =M_{y}^{2} \underbrace{\frac{\left(\epsilon_{k-1}-\epsilon_{k}\right)^{2}}{\epsilon_{k}^{3} \alpha_{k}(1-c)}\left(1+\frac{1}{\alpha_{k} \epsilon_{k}}\right)}_{\text {Term } 2} \\
& +\underbrace{\frac{\alpha_{k}}{\epsilon_{k}(1-c)} \mathbb{E}\left[\left\|w^{k}\right\|^{2} \mid \mathcal{F}_{k}\right]}_{\text {Term } 3}
\end{aligned}
$$

By (A3d) and (A3e), Terms 2 and 3 converge to zero in the limit, implying that $\lim _{k \rightarrow \infty} v_{k} / u_{k}=0$. Finally, also by $(\mathrm{A} 3 \mathrm{c})$ and $(\mathrm{A} 2 \mathrm{c})$, we have

$$
\sum_{k=1}^{\infty} v_{k}<\infty
$$

It follows from Lemma 1 that $\left\|x^{k}-y^{k-1}\right\|$ converges to 0 almost surely.

The probabilistic result follows directly from Lemma 1 .

Our next lemma ensures that a feasible set of steplength and regularization sequences satisfying the conditions of Assumption (A3) do indeed exist. 
Lemma 4: Consider the choice $\alpha_{k}=k^{-a}$ and $\epsilon_{k}=k^{-b}$ for all $k$, where $a \in(0,1), b \in(0,1), a+b<1, a>b$. Then, $\alpha_{k}$ and $\epsilon_{k}$ satisfy Assumption (A3).

Proof: The condition in (A3a) hold trivially for large $k$ because $a>b$. Also, (A3b) and (A3f) hold because $\alpha_{k} \epsilon_{k}=$ $k^{-a-b}>k^{-1}$. The limit in (A3e) follows from the fact that $\sum \alpha_{k}^{2} \mathbb{E}\left[\left\|w^{k}\right\|^{2} \mid \mathcal{F}_{k}\right]<\infty$ and $\sum \alpha_{k} \epsilon_{k}=\infty$. Under the given form of $\epsilon_{k}$ and $\alpha_{k}$ the equation in (A3c) becomes

$\frac{\left((k-1)^{-b}-k^{-b}\right)^{2}}{k^{-2 b}}\left(1+\frac{1}{k^{-a-b}}\right) \leq 2 \frac{\left((1-1 / k)^{-b}-1\right)^{2}}{k^{-a-b}}$,

where the inequality follows from the fact that

$$
\frac{1}{k^{-a-b}} \geq 1, \text { for } k \geq 1 \text {. }
$$

Using the expansion of $(1-x)^{-b}$ for $x$ small, we get

$$
\begin{aligned}
2 \frac{\left((1-1 / k)^{-b}-1\right)^{2}}{k^{-a-b}} & =2 \frac{\left(1+\frac{b}{k}+O\left(k^{-2}\right)-1\right)^{2}}{k^{-a-b}} \\
& \approx \frac{2 b^{2}}{k^{2-a-b}}+O\left(1 / k^{2}\right)<\frac{2}{k}
\end{aligned}
$$

where in the last inequality we use $a+b<1$. Following a similar argument, it can be verified that the term in (A2d) reduces to

$$
\begin{aligned}
& \frac{\left((k-1)^{-b}-k^{-b}\right)^{2}}{k^{-2 b}}\left(\frac{1}{k^{-a-b}}+\frac{1}{k^{-2 a-2 b}}\right) \\
& =\frac{b^{2}}{k^{2(1-a-b)}}+\frac{b^{2}}{k^{(2-a-b)}}
\end{aligned}
$$

and the limit in (A3d) follows from the fact that $a+b<1$.

\section{B. Limited Coordination of Steplengths}

In this subsection, we consider a situation when users have their individual stepsize and update their estimates according to the following rule:

$$
x_{i}^{k+1}=\Pi_{K_{i}}\left[x_{i}^{k}-\alpha_{k, i}\left(F_{i}\left(x^{k}\right)+w_{i}^{k}+\epsilon_{k} x_{i}^{k}\right)\right] .
$$

The rule is the same as in (5), except for the steplength $\alpha_{k}$, which is replaced with user $i$ stepsize $\alpha_{k, i}$.

In our analysis of the method in (12), we make use on the following assumptions on the user stepsizes and the regularization parameters.

Assumption 4 (A4): Suppose that:

(a) $\frac{\alpha_{k, \max }}{\alpha_{k, \min }} \frac{\alpha_{k, \max }}{\epsilon_{k}}\left(\epsilon_{0}+L\right)^{2} \leq c$ for all $k$ where $c \in(0,1)$;

(b) $0<\alpha_{k, \min } \epsilon_{k} \leq \frac{2}{1-c}$ for all $k$ and $\lim _{k \rightarrow 0} \epsilon_{k}=0$;

(c) $\sum_{k=1}^{\infty} \frac{\left(\epsilon_{k}-\epsilon_{k-1}\right)^{2}}{\epsilon_{k}^{2}}\left(1+\frac{1}{\alpha_{k, \min } \epsilon_{k}}\right)<\infty$;

(d) $\lim _{k \rightarrow \infty} \frac{\left(\epsilon_{k}-\epsilon_{k-1}\right)^{2}}{\epsilon_{k}^{3} \alpha_{k, \min }}\left(1+\frac{1}{\alpha_{k, \min \epsilon_{k}}}\right) \rightarrow 0$;

(e) $\lim _{k \rightarrow \infty} \frac{\alpha_{k, \text { max }}}{\epsilon_{k}} \mathbb{E}\left[\left\|w^{k}\right\|^{2} \mid \mathcal{F}_{k}\right]=0$;

(f) $\sum_{k=1}^{\infty} \alpha_{k, \min } \epsilon_{k}=\infty$;

(g) $1<\frac{\alpha_{k, \max }}{\alpha_{k, \min }}<1+\epsilon_{k} \frac{1-c}{4 L}$.

In the following proposition, we provide almost sure convergence result of the random sequence $\left\{x^{k}\right\}$.

Proposition 3: Let Assumptions (A2) and (A4) hold. Also, assume that $\operatorname{SOL}(K, F)$ is nonempty. Let the sequence $\left\{x^{k}\right\}$ be generated by method (12). Then, $\left\{x^{k}\right\}$ converges to the least norm solution $x^{*}$ of $\operatorname{VI}(K, F)$ almost surely.

Proof: By using the relation $y_{i}^{k}=\Pi_{K_{i}}\left[y_{i}^{k}-\right.$ $\left.\alpha_{k, i}\left(F_{i}\left(y^{k}\right)+\epsilon_{k} y_{i}^{k}\right)\right]$ and non-expansive property of the projection operator, it can be verified that

$$
\begin{aligned}
& \left\|x_{i}^{k+1}-y_{i}^{k}\right\|^{2} \\
& \leq\left\|x_{i}^{k}-y_{i}^{k}\right\|^{2}-2 \alpha_{k, i}\left(x_{i}^{k}-y_{i}^{k}\right)^{T}\left(F_{i}\left(x^{k}\right)-F_{i}\left(y^{k}\right)\right) \\
& -2 \alpha_{k, i} \epsilon_{k}\left\|x_{i}^{k}-y_{i}^{k}\right\|^{2}-2 \alpha_{k, i}\left(x_{i}^{k}-y_{i}^{k}\right)^{T} w_{i}^{k} \\
& +\alpha_{k, i}^{2}\left\|F_{i}\left(x^{k}\right)-F_{i}\left(y^{k}\right)+w_{i}^{k}+\epsilon_{k}\left(x_{i}^{k}-y_{i}^{k}\right)\right\|^{2} .
\end{aligned}
$$

The last term in the inequality can be expanded as

$$
\begin{aligned}
& \left\|F_{i}\left(x^{k}\right)-F_{i}\left(y^{k}\right)+w_{i}^{k}+\epsilon_{k}\left(x_{i}^{k}-y_{i}^{k}\right)\right\|^{2} \\
& =\left\|F_{i}\left(x^{k}\right)-F_{i}\left(y^{k}\right)\right\|^{2}+\left\|w_{i}^{k}\right\|^{2}+\epsilon_{k}^{2}\left\|x_{i}^{k}-y_{i}^{k}\right\|^{2} \\
& +2\left(F_{i}\left(x^{k}\right)-F_{i}\left(y^{k}\right)\right)^{T} w_{i}^{k}+2 \epsilon_{k}\left(x_{i}^{k}-y_{i}^{k}\right)^{T} w_{i}^{k} \\
& +2 \epsilon_{k}\left(F_{i}\left(x^{k}\right)-F_{i}\left(y^{k}\right)\right)^{T}\left(x_{i}^{k}-y_{i}^{k}\right) .
\end{aligned}
$$

Taking expectation and combining (13) and (14) together with $\mathbb{E}\left[w_{i}^{k} \mid \mathcal{F}_{k}\right]=0$ (cf. Assumption (A2c)) we get

$$
\begin{aligned}
& \mathbb{E}\left[\left\|x_{i}^{k+1}-y_{i}^{k}\right\|^{2} \mid \mathcal{F}_{k}\right] \\
& \leq\left(1-2 \alpha_{k, i} \epsilon_{k}+\alpha_{k, i}^{2} \epsilon_{k}^{2}\right)\left\|x_{i}^{k}-y_{i}^{k}\right\|^{2} \\
& +\alpha_{k, i}^{2}\left(\left\|F_{i}\left(x^{k}\right)-F_{i}\left(y^{k}\right)\right\|^{2}+\mathbb{E}\left[\left\|w_{i}^{k}\right\|^{2} \mid \mathcal{F}_{k}\right]\right) \\
& +2 \alpha_{k, i}^{2} \epsilon_{k}\left(x_{i}^{k}-y_{i}^{k}\right)^{T}\left(F_{i}\left(x^{k}\right)-F_{i}\left(y^{k}\right)\right) \\
& -2 \alpha_{k, i}\left(x_{i}^{k}-y_{i}^{k}\right)^{T}\left(F_{i}\left(x^{k}\right)-F_{i}\left(y^{k}\right)\right) .
\end{aligned}
$$

Summing over all $i$ and using $\alpha_{k, i} \leq \alpha_{k, \max }$ together with Lipschitz continuity of $F$ yields

$$
\begin{aligned}
& \mathbb{E}\left[\left\|x^{k+1}-y^{k}\right\|^{2} \mid \mathcal{F}_{k}\right] \\
& \leq\left(1-2 \alpha_{k, \min } \epsilon_{k}+\alpha_{k, \max }^{2} \epsilon_{k}^{2}+\alpha_{k, \max }^{2} L^{2}\right)\left\|x^{k}-y^{k}\right\|^{2} \\
& +\alpha_{k, \max }^{2} \mathbb{E}\left[\left\|w^{k}\right\|^{2} \mid \mathcal{F}_{k}\right] \\
& +\sum_{i=1}^{N} 2 \alpha_{k, i}^{2} \epsilon_{k}\left(x_{i}^{k}-y_{i}^{k}\right)^{T}\left(F_{i}\left(x^{k}\right)-F_{i}\left(y^{k}\right)\right) \\
& -\sum_{i=1}^{N} 2 \alpha_{k, i}\left(x_{i}^{k}-y_{i}^{k}\right)^{T}\left(F_{i}\left(x^{k}\right)-F_{i}\left(y^{k}\right)\right) .
\end{aligned}
$$

Next we estimate the inner product terms, $\left(x_{i}^{k}-\right.$ $\left.y_{i}^{k}\right)^{T}\left(F_{i}\left(x^{k}\right)-F_{i}\left(y^{k}\right)\right)$ of (15). The first term with $\alpha_{k, i}^{2} \epsilon_{k}$ can be estimated as follows:

$$
\begin{aligned}
& \sum_{i=1}^{N} 2 \alpha_{k, i}^{2} \epsilon_{k}\left(x_{i}^{k}-y_{i}^{k}\right)^{T}\left(F_{i}\left(x^{k}\right)-F_{i}\left(y^{k}\right)\right) \\
& \leq \alpha_{k, \max }^{2} \epsilon_{k} \sum_{i=1}^{N}\left\|x_{i}^{k}-y_{i}^{k}\right\|\left\|F_{i}\left(x^{k}\right)-F_{i}\left(y^{k}\right)\right\| .
\end{aligned}
$$

By Holder's inequality, we have $\sum_{i=1}^{N}\left\|x_{i}^{k}-y_{i}^{k}\right\| \| F_{i}\left(x^{k}\right)-$ $F_{i}\left(y^{k}\right)\|\leq\| x^{k}-y^{k}\|\| F\left(x^{k}\right)-F\left(y^{k}\right) \|$ which through the use of Lipschitz continuity of $F$ yields

$$
\begin{aligned}
& \sum_{i=1}^{N} 2 \alpha_{k, i}^{2} \epsilon_{k}\left(x_{i}^{k}-y_{i}^{k}\right)^{T}\left(F_{i}\left(x^{k}\right)-F_{i}\left(y^{k}\right)\right) \\
& \leq \alpha_{k, \max }^{2} \epsilon_{k} L\left\|x^{k}-y^{k}\right\|^{2} .
\end{aligned}
$$


Adding and subtracting the terms $\alpha_{k, \min }\left(x_{i}^{k}-y_{i}^{k}\right)^{T}\left(F_{i}\left(x^{k}\right)-\right.$ $\left.F_{i}\left(y^{k}\right)\right)$ to the second inner product term of (15) we have

$$
\begin{aligned}
& -\sum_{i=1}^{N} 2 \alpha_{k, i}\left(x_{i}^{k}-y_{i}^{k}\right)^{T}\left(F_{i}\left(x^{k}\right)-F_{i}\left(y^{k}\right)\right) \\
& \leq-2 \alpha_{k, \min }\left(x^{k}-y^{k}\right)^{T}\left(F\left(x^{k}\right)-F\left(y^{k}\right)\right) \\
& +2\left(\alpha_{k, \max }-\alpha_{k, \min }\right) \sum_{i=1}^{N}\left\|x_{i}^{k}-y_{i}^{k}\right\|\left\|F_{i}\left(x^{k}\right)-F_{i}\left(y^{k}\right)\right\| .
\end{aligned}
$$

Using monotonicity of $F$ we have $\left(x^{k}-y^{k}\right)^{T}\left(F\left(x^{k}\right)-\right.$ $\left.F\left(y^{k}\right)\right) \geq 0$. Further by the use of Holder's inequality and Lipschitz continuity of $F$, we get

$$
\sum_{i=1}^{N} 2 \alpha_{k, i}\left(x_{i}^{k}-y_{i}^{k}\right)^{T}\left(F_{i}\left(x^{k}\right)-F_{i}\left(y^{k}\right)\right) \leq 2 \delta_{k} L\left\|x^{k}-y^{k}\right\|^{2}
$$

where $\delta_{k} \triangleq \alpha_{k, \max }-\alpha_{k, \min }$. Combining the results of relation (15), (16) and (17) we obtain,

$$
\begin{aligned}
& \mathbb{E}\left[\left\|x^{k+1}-y^{k}\right\|^{2} \mid \mathcal{F}_{k}\right] \\
& \leq\left(1-2 \alpha_{k, \min } \epsilon_{k}+\alpha_{k, \max }^{2} \epsilon_{k}^{2}+\alpha_{k, \max }^{2} L^{2}\right)\left\|x^{k}-y^{k}\right\|^{2} \\
& +\alpha_{k, \max }^{2} \mathbb{E}\left[\left\|w^{k}\right\|^{2} \mid \mathcal{F}_{k}\right]+\left(2 \alpha_{k, \text { max }}^{2} \epsilon_{k} L+2 \delta_{k} L\right)\left\|x^{k}-y^{k}\right\|^{2} \\
& =q_{k}\left\|x^{k}-y^{k}\right\|^{2}+\alpha_{k, \text { max }}^{2} \mathbb{E}\left[\left\|w^{k}\right\|^{2} \mid \mathcal{F}_{k}\right],
\end{aligned}
$$

where $q_{k}:=1-2 \alpha_{k, \text { min }} \epsilon_{k}+\alpha_{k, \text { max }}^{2}\left(\epsilon_{k}+L\right)^{2}+2 \delta_{k} L$.

Next we invoke the estimate of $\left\|x^{k}-y^{k}\right\|^{2}$ from relation (11) but with a slight modification. Instead of using $\alpha_{k}$ in (10), we use $\alpha_{k, \min }$. Precisely, we have

$$
\begin{aligned}
& 2 \frac{\left|\epsilon_{k-1}-\epsilon_{k}\right|}{\epsilon_{k}}\left\|x^{k}-y^{k-1}\right\| M_{y} \\
= & 2 \sqrt{\alpha_{k, \min } \epsilon_{k}}\left\|x^{k}-y^{k-1}\right\| \frac{\left|\epsilon_{k-1}-\epsilon_{k}\right|}{\epsilon_{k} \sqrt{\alpha_{k, \min } \epsilon_{k}}} M_{y} \\
\leq & \alpha_{k, \min } \epsilon_{k}\left\|x^{k}-y^{k-1}\right\|^{2}+\frac{\left(\epsilon_{k-1}-\epsilon_{k}\right)^{2}}{\alpha_{k, \min } \epsilon_{k}^{3}} M_{y}^{2} .
\end{aligned}
$$

Using the modified estimate from (19) in earlier relation of (11) we obtain

$$
\begin{aligned}
\left\|x^{k}-y^{k}\right\|^{2} & \leq\left(1+\alpha_{k, \min } \epsilon_{k}\right)\left\|x^{k}-y^{k-1}\right\|^{2} \\
& +M_{y}^{2} \frac{\left(\epsilon_{k-1}-\epsilon_{k}\right)^{2}}{\epsilon_{k}^{2}}\left(1+\frac{1}{\alpha_{k, \min } \epsilon_{k}}\right) .
\end{aligned}
$$

Combining the relations of (18) and (20) we obtain the following estimate:

$$
\begin{aligned}
\mathbb{E}\left[\left\|x^{k+1}-y^{k}\right\|^{2}\right. & \left.\mid \mathcal{F}_{k}\right] \leq q_{k}\left(1+\alpha_{k, \min } \epsilon_{k}\right)\left\|x^{k}-y^{k-1}\right\|^{2} \\
& +q_{k} M_{y}^{2} \frac{\left(\epsilon_{k-1}-\epsilon_{k}\right)^{2}}{\epsilon_{k}^{2}}\left(1+\frac{1}{\alpha_{k, \min } \epsilon_{k}}\right) \\
& +\alpha_{k, \max }^{2} \mathbb{E}\left[\left\|w^{k}\right\|^{2} \mid \mathcal{F}_{k}\right] .
\end{aligned}
$$

Next we estimate the coefficient of $\left\|x^{k}-y^{k-1}\right\|^{2}$. Let $a_{k}:=$ $q_{k}\left(1+\alpha_{k, \min } \epsilon_{k}\right)$. Using $q_{k}<1$ (which holds by (A4a) and (A4g)) we see that

$$
\begin{aligned}
a_{k} & =q_{k}\left(1+\alpha_{k, \min } \epsilon_{k}\right) \\
& \leq q_{k}+\alpha_{k, \min } \epsilon_{k} \\
& =1-2 \alpha_{k, \min } \epsilon_{k}+\alpha_{k, \max }^{2}\left(\epsilon_{k}+L\right)^{2}+2 \delta_{k} L+\alpha_{k, \min } \epsilon_{k} .
\end{aligned}
$$

Using $\epsilon_{k} \leq \epsilon_{0}$ and

$$
\frac{\alpha_{k, \max }^{2}\left(\epsilon_{k}+L\right)^{2}}{\alpha_{k, \min } \epsilon_{k}} \leq \frac{\alpha_{k, \max }^{2}\left(\epsilon_{0}+L\right)^{2}}{\alpha_{k, \min } \epsilon_{k}} \leq c
$$

we obtain

$$
a_{k} \leq 1-\alpha_{k, \min } \epsilon_{k}\left(1-c-\frac{2 \delta_{k} L}{\alpha_{k, \min } \epsilon_{k}}\right) .
$$

From (A4g) we have

$$
\frac{2 \delta_{k} L}{\alpha_{k, \min } \epsilon_{k}} \leq \frac{1-c}{2} .
$$

Thus for $k$ large enough, (22) reduces to

$$
a_{k} \leq 1-\alpha_{k, \min } \epsilon_{k}\left(\frac{1-c}{2}\right)
$$

and (21) can be written compactly as:

$$
\mathbb{E}\left[\left\|x^{k+1}-y^{k}\right\|^{2} \mid \mathcal{F}_{k}\right] \leq\left(1-u_{k}\right)\left\|x^{k}-y^{k-1}\right\|^{2}+v_{k}
$$

where $u_{k} \triangleq \frac{1-c}{2} \alpha_{k, \min } \epsilon_{k}$ and

$v_{k}=q_{k} M_{y}^{2} \frac{\left(\epsilon_{k-1}-\epsilon_{k}\right)^{2}}{\epsilon_{k}^{2}}\left(1+\frac{1}{\alpha_{k, \min } \epsilon_{k}}\right)+\alpha_{k, \max }^{2} \mathbb{E}\left[\left\|w^{k}\right\|^{2} \mid \mathcal{F}_{k}\right]$.

Since $c<1$ we have $0 \leq u_{k} \leq 1$, and from (A4f) we have $\sum_{k=1}^{\infty} u_{k}=\infty$. Under stepsize condition (A4c), (A4d) and (A4e) it can be verified that

$$
\lim _{k \rightarrow \infty} \frac{v_{k}}{u_{k}}=0 \quad \text { and } \quad \sum_{k=1}^{\infty} v_{k}<\infty .
$$

Thus, the conditions of Lemma 1 are satisfied for $k$ large enough. Noting that Lemma 1 applies to a process delayed by a deterministic time-offset, we can conclude that $\left\|x^{k}-y^{k-1}\right\|$ converges to 0 almost surely. This and the fact that $y^{k} \rightarrow x^{*}$ conclude the proof.

\section{CONCLUDING REMARKS}

This paper is motivated by the need to compute equilibria associated with stochastic Nash games when the mappings did not display a desirable strong monotonicity property. Past work by Jiang and $\mathrm{Xu}$ [12] considered how stochastic approximation procedures could address stochastic variational inequalities when the mappings were strongly monotone. Yet, these schemes could not easily contend with weaker requirements (such as strict monotonicity or merely monotonicity) while retaining the single-timescale structure. Instead, a simple regularization-based extension would lead to a two timescale method, that is generally harder to implement in networked settings.

Accordingly, this paper makes two contributions. First, we show that a standard stochastic approximation scheme is shown to lead to almost-sure convergence under the assumption of strict monotonicity. Second, when the assumption on the mapping is further weakened to monotone, then we turn to a class of iterative Tikhonov regularization schemes. A crucial difference that such methods display with their classical Tikhonov counterpart is that the regularization parameter is updated at every iteration. An extension of this 
framework is proposed to the stochastic regime and suitable conditions are established for the almost-sure convergence of the resulting scheme. These results require that the steplengths are common across players. We further show that when this requirement is relaxed to one where steplengths are partially coordinated across players, as is the need in networked settings, convergence of the scheme still holds.

\section{REFERENCES}

[1] T. Alpcan and T. Başar, A game-theoretic framework for congestion control in general topology networks, 41th IEEE Conf. Decision and Control (Las Vegas, NV), December 2002.

[2] _ Distributed algorithms for Nash equilibria of flow control games, Advances in Dynamic Games, Annals of the International Society of Dynamic Games, vol. 7, Birkhäuser Boston, 2003, pp. 473498.

[3] E. Altman, T. Basar, T. Jiménez, and N. Shimkin, Competitive routing in networks with polynomial cost, INFOCOM (3), 2000, pp. 15861593.

[4] D.P. Bertsekas and J.N. Tsitsiklis, Gradient convergence in gradient methods, SIAM Journal on Optimization 10 (2000), no. 3, 627-642.

[5] M. Bloem, T. Alpcan, and T. Başar, A stackelberg game for power control and channel allocation in cognitive radio networks, ValueTools '07: Proceedings of the 2nd international conference on Performance evaluation methodologies and tools (ICST, Brussels, Belgium, Belgium), ICST (Institute for Computer Sciences, Social-Informatics and Telecommunications Engineering), 2007, pp. 1-9.

[6] V.S. Borkar, Stochastic approximation: A dynamical systems viewpoint, Cambridge University Press, 2008.

[7] D. Dutta, A. Goel, and J. Heidemann, Oblivious aqm and nash equilibria, INFOCOM 2003. Twenty-Second Annual Joint Conference of the IEEE Computer and Communications Societies. IEEE 1 (2003), $106-113$ vol.1.

[8] Y. Ermoliev, Stochastic programming methods, Nauka, Moscow, 1976.

[9] - Stochastic quasi-gradient methods and their application to system optimization, Stochastics 9 (1983), no. 1, 1-36.

[10] _ Stochastic quazigradient methods, Numerical Techniques for Stochastic Optimization, Springer-Verlag, N.Y., 1988, pp. 141-186.

[11] F. Facchinei and J-S. Pang, Finite-dimensional variational inequalities and complementarity problems, vol. I and II, Springer-Verlag New York, 2003.

[12] H. Jiang and H. Xu, Stochastic approximation approaches to the stochastic variational inequality problem, Automatic Control, IEEE Transactions on 53 (2008), no. 6, 1462-1475.

[13] I. V. Konnov, Equilibrium models and variational inequalities, Mathematics in Science and Engineering, vol. 210, Elsevier, Amsterdam, 2007.

[14] Y. Pan and L. Pavel, Games with coupled propagated constraints in optical network with multi-link topologies, Automatica 45 (2009), 871-880.

[15] L. Pavel, A noncooperative game approach to OSNR optimization in optical networks, IEEE Transactions on Automatic Control 51 (2006), no. $5,848-852$.

[16] L. Pavel, An extension of duality to a game-theoretic framework, Automatica 43 (2007), 226-237.

[17] B.T. Polyak, Introduction to optimisation, Optimization Software, Inc., New York, 1987.

[18] S. Sundhar Ram, A. Nedić, and V.V. Veeravalli, Incremental stochastic sub-gradient algorithms for convex optimization, SIAM Journal on Optimization 20 (2009), no. 2, 691-717.

[19] A. Rantzer, Using game theory for distributed control engineering, Tech. Report ISRN LUTFD2/TFRT--7620--SE, Department of Automatic Control, Lund University, Sweden, July 2008, Presented at Games 2008, 3rd World Congress of the Game Theory Society.

[20] H. Robbins and S. Monro, A stochastic approximation method, The Annals of Mathematical Statistics 22 (1951), no. 3, 400-407.

[21] H. Yin, U.V. Shanbhag, and P.G. Mehta, Nash equilibrium problems with congestion costs and sharedconstraints, Under revision (2009). 